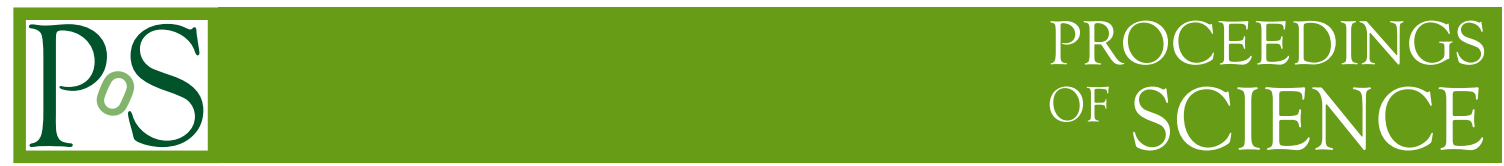

Measurements of CP violation in the $B_{s}$ system at D0

\title{
Sergey BURDIN*i
}

University of Liverpool

E-mail: burdineliv.ac.uk

An updated measurement of the CP-violating phase $\phi_{s}^{J / \psi \phi}$ and measurement of the relative branching ratio of $B_{s} \rightarrow J / \psi f_{0}$ to $B_{s} \rightarrow J / \psi \phi$ using data samples corresponding to an integrated luminosity of $8 \mathrm{fb}^{-1}$ recorded with the D0 detector are reported.

The 2011 Europhysics Conference on High Energy Physics-HEP 2011,

July 21-27, 2011

Grenoble, Rhône-Alpes France

\footnotetext{
* Speaker.

${ }^{\dagger}$ For D0 Collaboration.
} 


\section{Introduction}

One of the most intriguing Tevatron results is related to the charge-conjugation-parity (CP) violation measurements in the $B_{s}$ system. Both CDF and D0 experiments [1], 2] observed deviations from the Standard Model predictions for the CP-violating phase $\left(\phi_{s}^{J / \psi \phi}\right)$ which is measured in the $B_{s} \rightarrow J / \psi \phi$ decays. Though the deviations are not statistically significant, they agree with each other and with the measurement of the $\mathrm{CP}$ violation in the $B_{s}$ system using same-sign dimuon events [3]. In the Standard Model (SM) the decay $B_{s} \rightarrow J / \psi \phi$ goes through the decay of the $b$-quark to $c \bar{c} s$ combination, and the value of $\phi_{s}^{J / \psi \phi}$ is proportional to the angle $\beta_{s}$ of the $B_{s}$ Unitarity Triangle derived from the Cabibbo-Kobayashi-Maskawa quark-mixing matrix [ [ $\llbracket$ ] and determined by the interference of the decays with and without $B_{s}-\bar{B}_{s}$ mixing:

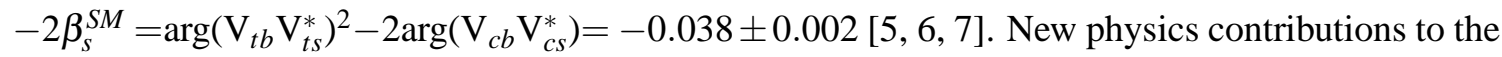
mixing could change the phase and be observed by measuring strong deviation from the predicted value: $\phi_{s}^{J / \psi \phi}=-2 \beta_{s}^{S M}+\phi_{N P} \approx \phi_{N P}$. The measurement of $\phi_{s}^{J / \psi \phi}$ in the $B_{s} \rightarrow J / \psi \phi$ channel requires disentangling the CP-odd and CP-even final-decay states because both $J / \psi$ and $\phi$ are vector mesons. The $\mathrm{CP}$ eigenstates have different angular distributions, masses $\left(\Delta M_{s}=M_{H}-M_{L}\right)$ and decay widths $\left(\Delta \Gamma_{s}=\Gamma_{L}-\Gamma_{H}\right)$ assuming mass eigenstates are pure CP eigenstates. Measurements of the CP-violating phase $\phi_{s}^{J / \psi \phi}$ depend on $\Delta \Gamma_{s}$, and the results are often presented as contours in the $\left(\phi_{s}^{J / \psi \phi}, \Delta \Gamma_{s}\right)$ plot. An additional complication in these measurements appears due to the $\sim 10 \%$ non-resonant $S$-wave contribution under the $\phi$ peak (Fig. 1) [8] which introduces unknown fraction $\left(F_{S}\right)$ and phase $\left(\delta_{s}\right)$ into the analysis.

The $\phi_{s}^{J / \psi \phi}$ measurement is more straightforward in $B_{s} \rightarrow J / \psi f_{0}$ decays due to the presence of the CP-odd final state only ( $f_{0}$ meson is a scalar) [5] but the branching ratio $\mathscr{B}\left(B_{s} \rightarrow \boldsymbol{J} / \psi f_{0}, f_{0} \rightarrow\right.$ $\left.\pi^{+} \pi^{-}\right)$is approximately four times less than $\mathscr{B}\left(B_{s} \rightarrow J / \psi \phi, \phi \rightarrow K^{+} K^{-}\right)$. Precise determination of this ratio is the first step towards CP violation measurements using the $B_{s} \rightarrow J / \psi f_{0}$ channel.

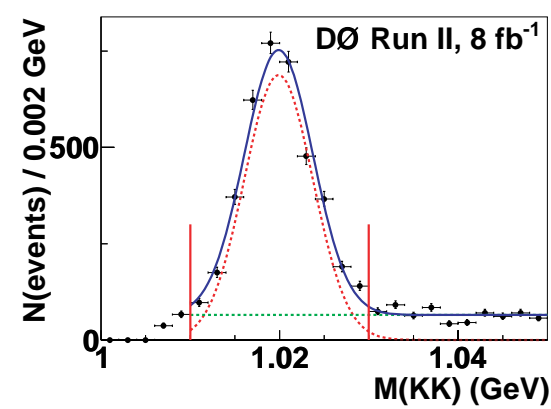

Figure 1: The invariant mass distribution of two kaon candidates when the $J / \psi K^{+} K^{-}$invariant mass is consistent with the $B_{S}$ mass. The solid line is a fit to a sum of the $P$-wave $\phi(1020)$ meson decay and a constant term due to $S$ wave.

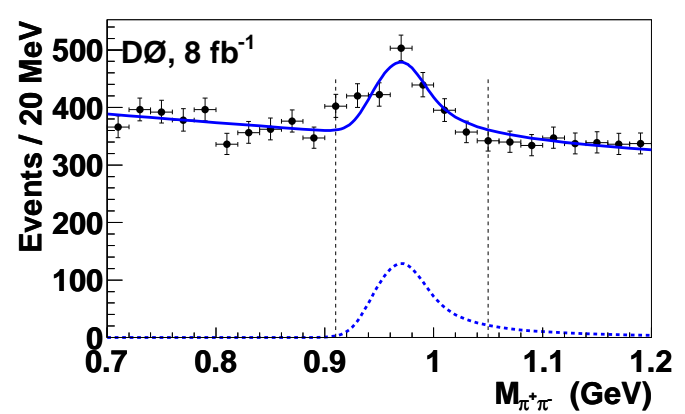

Figure 2: The invariant mass distribution of two pion candidates when the $J / \psi \pi^{+} \pi^{-}$invariant mass is consistent with the $B_{S}$ mass. The dashed line is the fitted $f_{0}(980)$ signal.

\section{Event Selection}

The signal event candidates for the $B_{s} \rightarrow J / \psi \phi$ and $B_{s} \rightarrow J / \psi f_{0}$ channels were selected using 
the Boosted Decision Tree (BDT) algorithm. The $J / \psi \rightarrow \mu^{+} \mu^{-}$decays give a clean sample of $J / \psi$ candidates. The signal candidates are constructed by adding two oppositely charged tracks which create a common vertex with the muons and have an invariant mass consistent with the $\phi\left(f_{0}\right)$ meson in kaon (pion) hypothesis (Figs. 1, 2). The BDT algorithms were trained on the Monte Carlo (MC) signal and background samples for background events suppression. Two types of background were considered separately: prompt $(J / \psi$ mesons are produced directly) and non-prompt $(J / \psi$ mesons are produced in decays of $b$-quarks).

The selections of $B_{s} \rightarrow J / \psi \phi, \phi \rightarrow K^{+} K^{-}$candidates in case of the relative branching ratio measurement of $B_{s} \rightarrow J / \psi f_{0}$ were fixed to be the same as for $B_{s} \rightarrow J / \psi f_{0}, f_{0} \rightarrow \pi^{+} \pi^{-}$candidates except for the $f_{0}(980)$ mass requirement.

The selections of $B_{s} \rightarrow J / \psi \phi, \phi \rightarrow K^{+} K^{-}$candidates for the CP violation measurement were tuned to minimize statistical uncertainties on $\phi_{s}^{J / \psi \phi}$ and $\Delta \Gamma_{s}$. Simple selection criteria [9] were utilized for a second sample which has been used for consistency checks and systematic effects studies.

\section{Measurement of the relative branching ratio of $B_{s} \rightarrow J / \psi f_{0}$ to $B_{s} \rightarrow J / \psi \phi$}

The measurement of the relative branching ratio $R_{f_{0} / \phi}=\frac{N_{B_{s} \rightarrow J / \psi} f_{0}}{N_{B_{S} \rightarrow J / \psi}} \cdot \frac{\varepsilon_{r c c o}^{B} \rightarrow J / \psi \phi}{\varepsilon_{r e c} \rightarrow J / \psi f_{0}}$ requires knowledge of the corresponding event yields and reconstruction efficiencies. Detailed description of the analysis is given in [10]. The numbers of $B_{s}$ events determined from the $J / \psi f_{0}$ and $J / \psi \phi$ mass distributions are $590 \pm 84$ and $2929 \pm 62$ respectively. The $B_{s} \rightarrow J / \psi \phi$ yield should be scaled by the factor 0.88 [ [ ] the take into account the $S$-wave contribution. The mean relative reconstruction efficiency determined from MC samples and averaged over all the running periods is $\frac{\varepsilon_{\text {reco }}^{B_{s} \rightarrow J / \psi \phi}}{\mathcal{E}_{\text {reco }} \rightarrow J / \psi f_{0}}=1.20 \pm 0.04$.

The resulting relative branching ratio is $R_{f_{0} / \phi}=0.275 \pm 0.041$ (stat) \pm 0.061 (syst) with the systematic uncertainties originating from fitting $(17.3 \%)$, MC efficiency $(9.2 \%)$, modeling variables in BDT (8.9\%), $f_{0}(980)$ mass window $(4.0 \%)$ and $S$-wave contibution $(3.5 \%)$.

\section{CP violation measurements in $B_{S} \rightarrow J / \psi \phi$}

The angular and decay time distributions used in the $B_{s} \rightarrow J / \psi \phi$ analysis are described in [2]. The following expression illustrates the asymmetry of the $B_{S} / \bar{B}_{S}$ decay rates [1]]: $\frac{P_{ \pm}(t)-\bar{P}_{ \pm}(t)}{P_{ \pm}(t)+\bar{P}_{ \pm}(t)}=$ $\pm\left[\frac{2 \sin \left(\Delta M_{s} t\right) \sin \phi_{s}^{J / \psi \phi}}{\left(1 \pm \cos \phi_{s}^{J / \psi \phi}\right) e^{+\Delta \Gamma_{s} t / 2}+\left(1 \mp \cos \phi_{s}^{J / \psi \phi}\right) e^{-\Delta \Gamma_{s} t / 2}}\right]$, where $P_{ \pm}(t)$ and $\bar{P}_{ \pm}(t)$ refer to CP-even $(+)$ and CPodd (-) final states tagged as $B_{s}$ or $\bar{B}_{s}$ at the production time.

Numbers of $B_{s} \rightarrow J / \psi \phi$ candidates were determined from mass fits to be $5598 \pm 113$ in case of the BDT selections and $5050 \pm 105$ in case of the simple selections. The analysis results are following: $\bar{\tau}_{s}=1.443_{-0.035}^{+0.038} \mathrm{ps}, \Delta \Gamma_{s}=0.163_{-0.064}^{+0.065} \mathrm{ps}^{-1}, \phi_{s}^{J / \psi \phi}=-0.55_{-0.36}^{+0.38},\left|A_{0}\right|^{2}=0.558_{-0.019}^{+0.017}$, $\left|A_{\|}\right|^{2}=0.231_{-0.030}^{+0.024}, \delta_{\|}=3.15 \pm 0.22, \cos \left(\delta_{\perp}-\delta_{s}\right)=-0.11_{-0.25}^{+0.27}, F_{S}=0.173 \pm 0.036$. Some parameters are not defined in this report but full description is available in [2]. Systematic uncertainties were determined using Markov Chain Monte Carlo technique and combined with statistical uncertainties. Two-dimensional 68\%, 90\% and 95\% Confidence Level (C.L.) contours are shown in Fig. 3. The $p$-value for the SM point $\left(\phi_{s}^{J / \psi \phi}, \Delta \Gamma_{s}\right)=\left(-0.038,0.087 \mathrm{ps}^{-1}\right)$ is $29.8 \%$. 


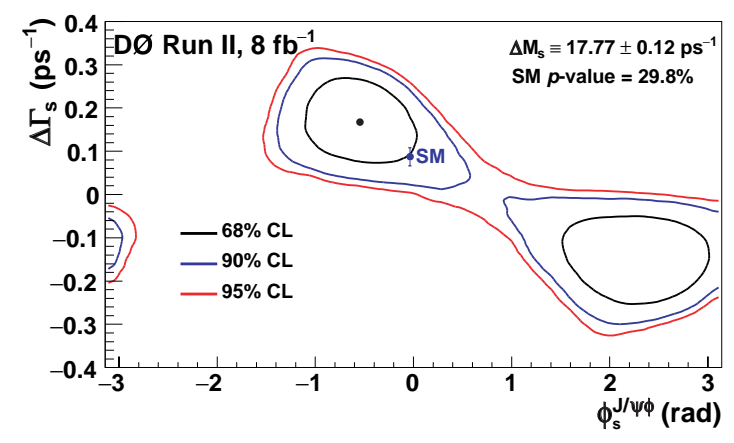

Figure 3: Two-dimensional $68 \%, 90 \%$ and $95 \%$ C.L. contours including systematic uncertainties. The standard model expectation is indicated as a point with an error.

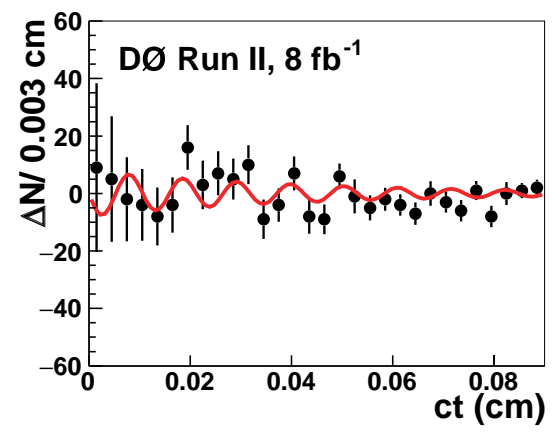

Figure 4: Proper decay length evolution of the difference $\Delta N=N\left(B_{S}\right)-N\left(\bar{B}_{S}\right)$ in the first $0.09 \mathrm{~cm}$ (3 ps) for the simple selection sample. The curve represents the best fit to the oscillation with the frequency of $\Delta M_{S}=17.77 \mathrm{ps}^{-1}$.

The asymmetry equation above shows that the time-dependent decay rates for $B_{s}$ and $\bar{B}_{s}$ are not equal given non-zero $\phi_{s}^{J / \psi \phi}$. The time-dependent asymmetry can be represented as $\Delta N \equiv$ $N\left(B_{S}\right)-N\left(\bar{B}_{s}\right)=N_{S} \cdot C \cdot \sin \left(\phi_{s}^{J / \psi \phi}\right) \cdot \sin \left(\Delta M_{s} t\right) \cdot e^{-t / \tau_{s}}$, where $N_{S}$ is number of $B_{s}$ and $\bar{B}_{s}$ candidates and $C \approx 0.0025$ is a dilution factor. Fig. 7 shows the proper decay length evolution of $\Delta N$ in the first $900 \mu \mathrm{m}\left(\sim 2 c \bar{\tau}_{S}\right)$ for the simple selection sample. The fit gives $N_{S} \cdot C \cdot \sin \left(\phi_{S}^{J / \psi \phi}\right)=-8 \pm 4$ which is consistent with expectations from the $\phi_{S}^{J / \psi \phi}=-0.55_{-0.36}^{+0.38}$ result.

\section{Conclusion}

This report presents short description of two D0 analyses: the measurement of the relative branching ratio of $B_{s} \rightarrow J / \psi f_{0}$ and CP violation measurements in $B_{s} \rightarrow J / \psi \phi$. The used data samples correspond to $8 \mathrm{fb}^{-1}$. The $B_{s} \rightarrow J / \psi \phi$ results supersede the previous D0 measurements.

\section{Acknowledgments}

We thank the staffs at Fermilab and collaborating institutions, and acknowledge support from the DOE and NSF (USA); CEA and CNRS/IN2P3 (France); FASI, Rosatom and RFBR (Russia); CNPq, FAPERJ, FAPESP and FUNDUNESP (Brazil); DAE and DST (India); Colciencias (Colombia); CONACyT (Mexico); KRF and KOSEF (Korea); CONICET and UBACyT (Argentina); FOM (The Netherlands); STFC and the Royal Society (United Kingdom); MSMT and GACR (Czech Republic); CRC Program and NSERC (Canada); BMBF and DFG (Germany); SFI (Ireland); The Swedish Research Council (Sweden); and CAS and CNSF (China).

\section{References}

[1] CDF Collaboration, CDF note 10206.

[2] D0 Collaboration, arXiv:1109.3166.

[3] D0 Collaboration, Phys. Rev. D84 (2011) 052007. 
[4] M. Kobayashi and T. Maskawa, Prog. Theor. Phys. 49 (1973) 652.

[5] Isard Dunietz, et al., Phys. Rev. D63 (2001) 114015.

[6] A. Lenz and U. Nierste, J. High Energy Phys. 06 (2007) 072.

[7] M. Bona, et al., J. High Energy Phys. 10 (2006) 081.

[8] S. Stone and L. Zhang, Phys. Rev. D79 (2009) 074024.

[9] D0 Collaboration, Phys. Rev. Lett. 98 (2007) 121801.

[10] D0 Collaboration, arXiv:1110.4272.

[11] R. Fleischer, in proceedings of HQL2006, ECONF C0610161 (2006) 020 [hep-ph/0703112]. 